\title{
GW23-e0831 STUDY OF LEFT VENTRICULAR ROTATION AND TORSION IN HYPERTHYROID BY SPECKLE TRACKING IMAGING
}

doi:10.1136/heartjnl-2012-302920a.87

Zhao-jun Li, Chun-song Kang, Zhexia Zhao, Ji-ping Xue, Kai-ling Shi, Chun-song Kang. Department of Ultrasound, Shanxi Academy of Medical Sciences \& Shanxi DAYI Hospital

Objectives Quantitative analysis of hyperthyroidism in patients with left ventricular rotation and torsion motion by speckle tracking imaging (STI) to explore the hyperthyroidism in patients with left ventricular systolic function.

Methods Select the 59 cases hyperthyroidism who first $\mathrm{I}^{131}$ treatment and review cure and improvement after 6 month for the study, divided into two groups according to duration. Group A: duration of less than 6 months, 31 cases; B: duration of greater than 6 months, 28 cases; Control group: 30 cases of sex and age matched normal .Group A and B before treatment and after treatment with the control group underwent routine ultrasound examination, collection and storage of the left ventricular apex level of short-axis dynamic images of the cardiac cycle in all three, 
application X-strain software analysis come to the heart of the heart and the apex level, the rotation angle of sub-epicardial myocardial peak, calculation of left ventricular heart, sub-epicardial myocardial reverse angle peak, analysis the relativities between the rotation and torsion angle peak in cardiac structure and function parameters, heart rate, blood pressure and thyroid hormone levels and other factors .

\section{Results}

(1) Group A, B before and after treatment compared with the control group, left ventricular structure and function parameters (LVDd, LVDs, IVSd, PWd, LVEF), the difference was not statistically significant ( $p>0.05$ ), B group before treatment, left ventricular outflow tract velocity (LVOT-V) higher than that of the control group $(p<0.01)$ after treatment compared with control group difference not statistically significant $(p>0.05)$.

(2) The apex level of the heart, sub-epicardial myocardial rotation angle peak (EN-PAR, the EP-PAR) compared with Group A before treatment EN-PAR, the EP-PAR higher than that in the control group, EN-PAR, EP-PAR of Group B is lower than control group (EN: $6.01 \pm 2.54$ vs $5.18 \pm 2.17$ vs $4.38 \pm 2.46$ vs; EP: $2.31 \pm 1.06$ vs $1.87 \pm 1.04$ vs $1.54 \pm 0.78, p<0.01)$; after treatment, EN-PAR, EPPAR of group A than before treatment to improve B only EP-PAR than before treatment improved (Group A: EN:5.27 \pm 2.11 vs 6.01 \pm 2.54 ; EP: $1.87 \pm 1.04$ vs $2.31 \pm 1.06$, Group B: EN:4.81 \pm 2.17 vs 4.38 \pm 2.46 ; EP: $1.77 \pm 1.01$ vs $1.54 \pm 1.01, \mathrm{p}<0.01$ ).

(3) The heart of global left ventricular sub-epicardial myocardial reverse angle peak (EN-Ptor, EP-Ptor) comparison of Group A before treatment EN-Ptor, EP-Ptor higher than that in the control group, B EN-Ptor, EP-Ptor lower the control group (EN: 11.18 \pm 4.04 vs $9.53 \pm 2.69$ vs $7.77 \pm 3.35$; EP: $4.46 \pm 1.58$ vs $3.86 \pm 1.22$ vs $2.97 \pm 1.11, \mathrm{p}<0.01)$; after treatment, EN-Ptor, EP-Ptor of A and B improved (Group A: EN:9.83 $\pm 2.60 v s 11.18 \pm 4.04$; EP:3.90 \pm 1.20 vs 4.46 \pm 1.58 , Group B: EN: $9.01 \pm 2.69$ vs $7.77 \pm 3.35$; EP: $3.93 \pm 1.11$ vs $2.97 \pm 1.12, p<0.05)$ than before treatment.

(4) The level of the heart within the heart, sub-epicardial myocardial rotation angle peak (EN-PBR, the EP-PBR) Comparison of the B group before treatment and after treatment EN-of PBR, the EP-of PBR in the control group, no statistically significant difference $(p>0.05)$

(5) The apex of the heart, sub-epicardial myocardial rotation angle peak SBP, HR, LVOT-V showed a negative correlation $(p<0.01)$, and thyroid hormone levels (FT3, FT4 and TSH) no correlation $(\mathrm{p}>0.05)$.

\section{Conclusions}

(1) Hyperthyroidism in patients with early heart apex level, the adventitia of myocardial rotation and left ventricular peak torsion angle increases, decreases with the extension of the course.

(2) Has not yet appeared hyperthyroid heart disease, hyperthyroidism in patients with early left ventricular systolic function enhanced with the extension of the course to reduce.

(3) Short duration of the hyperthyroid patients after treatment, left ventricular regional systolic function of recoverability, the duration of the elderly cannot be fully restored.

(4) Change of the rotational motion of hyperthyroidism in patients with myocardial and hemodynamic changes in.

(5) STI can evaluate regional myocardial systolic function in patients with hyperthyroidism. 